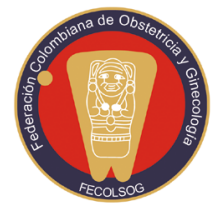

Revista Colombiana de Obstetricia y Ginecología Vol. 71 No. 2 • Abril-Junio 2020 • (178-194)

\title{
ASPECTOS SOCIALES QUE HAN AFECTADO LA ACEPTACIÓN DE LA VACUNACIÓN CONTRA EL VIRUS DEL PAPILOMA HUMANO EN COLOMBIA. UNA REVISIÓN SISTEMÁTICA
}

\section{Social considerations affecting acceptance of HPV vaccination in Colombia. A systematic review}

Francisco Palencia-Sánchez, MD, MSc, PhD (c) ${ }^{1}$; Sandra Johanna Echeverry-Coral, $M_{S c^{2}}$

Recibido: 21 de noviembre de 2019 /Aceptado: 04 de mayo de 2020

\section{RESUMEN}

Objetivo: establecer los aspectos sociales que afectaron la aceptación de la vacuna del virus del papiloma humano (VPH) en la población colombiana antes y después del evento mal definido e inusitado de etiología desconocida sucedido en 2014.

Materiales y métodos: se realizó una búsqueda sistemática de literatura en las bases de datos: Scopus, Web of Science, Medline vía PubMed, Embase, Biblioteca Virtual en Salud y Ovid; además, en Google Académico y en repositorios de universidades y en instituciones de salud en Colombia, con los términos: "Vacuna Tetravalente Recombinante contra el Virus del Papiloma Humano Tipos 6, 11, 16, 18”, “Colombia", "Papiloma” y sus correspondientes términos en inglés, para identificar principalmente revisiones sistemáticas, estudios cuantitativos y cualitativos, y revisiones narrativas que se enfocaran en aspectos sociales como:

* Correspondencia: Francisco Palencia-Sánchez, carrera 7, \# 40-62, Edificio Hospital San Ignacio, piso 8. fpalencia@javeriana.edu.co MSc Epidemiología Clínica, PhD (c) Salud Pública. Departamento de Medicina Preventiva y Social, Facultad de Medicina, Pontificia Universidad Javeriana, Bogotá (Colombia).

Bacterióloga, MSc en Epidemiología. Consultora Independiente, Bogotá (Colombia). educación, acceso, relación con el personal de salud, papel de los medios de comunicación, que pudieran haber actuado como barreras o facilitadores para la aceptación de vacunación para VPH en Colombia en el periodo 2006-2018. Se hace una síntesis narrativa de la información.

Resultados: se incluyeron 24 documentos. Se identificó la importancia, para los padres, adolescentes, proveedores y los medios, de tener un mejor conocimiento del VPH y su relación con el cáncer de cuello uterino (CCU). Se reconoce la relevancia de una buena comunicación entre las profesiones de la salud y la comunidad para informar adecuadamente tanto los beneficios como los riesgos de la vacuna. La inclusión en los planes de aseguramiento facilitó el acceso a esta por parte de la población. Los medios de comunicación deben ser considerados para que actúen como facilitadores de los programas de vacunación.

Conclusión: educar en el conocimiento del VPH, una atención en salud centrada en el paciente y una adecuada cobertura de los medios de comunicación influencian la aceptación del programa de vacunación contra VPH en la población colombiana. Se requiere seguir haciendo seguimiento estricto de los efectos adversos asociados a la vacuna. 
Palabras clave: vacuna tetravalente recombinante contra el virus del papiloma humano tipos 6, 11, 16, 18; Colombia; papiloma.

\section{ABSTRACT}

Objective: To identify social factors influencing the acceptance of human papilloma virus (HPV) vaccination in the Colombian population before and after the unexpected and poorly defined event of unknown etiology which occurred in 2014.

Materials and methods: A systematic review of the literature was conducted in the following databases: Scopus, Web of Science, Medline via PubMed, Embase, Online Health Library (Biblioteca Virtual en Salud) and Ovid, and also in Google Scholar, academic repositories and in Colombian health institutions, using the terms "recombinant tetravalent vaccine against Human Papilloma Virus types 6, 11, 16, 18”, "Colombia", "Papilloma" in order to primarily identify systematic reviews, quantitative and qualitative studies, narrative reviews, focusing on social aspects such as education, access, relationship with healthcare staff and role of the media which may have acted as barriers or facilitators for the acceptance of HPV vaccination in Colombia between 2006-2018. A narrative synthesis of the data was made.

Results: Twenty-four documents were included. The importance attached by parents, adolescents, providers and the media to having greater knowledge about HPV and its association with cervical cancer was identified. The relevance of good communication among healthcare professions and the community to enable adequate sharing of information regarding the risks and benefits of the vaccines was recognized. The inclusion of the vaccine in health insurance plans made access easier. The media must be involved as facilitators in vaccination programs.

Conclusion: Education regarding HPV, patientcentered healthcare and adequate media coverage influence the acceptance of HPV vaccination in the Colombian population. Close follow-up of any vaccine-related adverse events is required.

Key words: Recombinant tetravalent vaccine against human papilloma virus types $6,11,16,18$; Colombia; papiloma.

\section{INTRODUCCIÓN}

El virus del papiloma humano (VPH) produce la infección viral transmitida por contacto sexual más común en el tracto reproductivo de acuerdo con la Organización Mundial de la Salud (OMS) (1). Los serotipos 16 y 18 del VPH causan lesiones que pueden progresar a cáncer cervical (CC) si no son tratadas (2). Por otra parte, también está asociado al desarrollo de otros tipos de cáncer: se ha descrito que la fracción atribuible al VPH en el cáncer de pene es del 50\%, en el cáncer de vulva representa el $43 \%$, en cáncer de vagina el $70 \%$ y de ano el $88 \%$ (3).

A nivel mundial, el cáncer de cuello uterino (CCU) es el cuarto tipo de cáncer más común en mujeres (4). La incidencia de CCU varía ampliamente entre países, es el cáncer más frecuente en mujeres en 39 países, y una de las principales causas de muerte en 45 naciones, principalmente en África subsahariana, partes de Asia y algunos países de Centro y Sur América (5-7).

Desde hace 30 años la prevalencia de CCU ha disminuido, esta reducción posiblemente está relacionada con la mejoría de las condiciones de vida y la implementación de programas efectivos de prevención primaria como, por ejemplo, la estrategia de implementar planes masivos de tamización del cáncer de cuello con citología cérvico-vaginal (7). En Colombia, el cáncer de cérvix ha disminuido su frecuencia durante los últimos 40 años, según el registro poblacional de cáncer de Cali (8); sin embargo, se ha informado que entre los años 2012 a 2016, este tipo de cáncer fue la segunda causa de cáncer en mujeres $(7,1 \%)$ y la quinta causa de mortalidad para el periodo 2014 a 2018 (6,6\%); por lo que sigue siendo un problema de salud pública (9). 
Para marzo de 2017, el $37 \%$ de los países a nivel mundial habían introducido la vacuna contra el VPH en los planes de inmunización para niñas y el 6\% también lo hizo para población de sexo masculino (10). Colombia fue uno de los países pioneros al introducir la vacunación contra el VPH en agosto de 2012, con el uso de vacuna en niñas de 9 años de edad (11). Luego, mediante la Ley 1626 de 30 de abril de 2013, se garantizó la vacunación gratuita al ser incluida la vacuna contra el VPH en el Programa Ampliado de Inmunización (PAI) (12). En 2014, Colombia se consolidó como uno de los países a nivel mundial en contar con las más altas tasas de cobertura de la población objeto del programa de vacunación contra el VPH (13). La evaluación de la primera fase de vacunación en 2012 mostró un total de 1.015.405 dosis aplicadas a nivel nacional, y alcanzó un porcentaje de cobertura del $98 \%$ en la primera dosis, 96,9\% para la segunda y $89,2 \%$ para la tercera. En 19 de los 36 departamentos de Colombia, las coberturas fueron del $100 \%$ en la población objetivo. Aunque los porcentajes de cobertura bajaron al 87,6\% para la primera dosis de la vacuna, en la segunda fase (2013) se aplicó un total de 4.006.426 dosis a nivel nacional y el $53 \%$ de los departamentos seguía presentando cifras de cobertura superiores al $70 \%$ (14).

Desde el año 2014, a raíz de los sucesos en Carmen de Bolívar, en los que tras la aplicación de la vacuna contra el VPH se reportaron más de 500 casos de eventos supuestamente atribuidos a la vacunación o a la inmunización (ESAVI) (15), se redujeron las tasas de vacunación a 203.276 dosis a nivel nacional, con una cobertura del 63,6\% para primera dosis, y de 1,4\% para la segunda dosis al finalizar el año de la ocurrencia del evento descrito, tal como lo señalan algunos estudios elaborados posteriormente al evento (16).

No se conocen bien los factores socioculturales que pudieron asociarse a la reducción de la tasa de vacunación. Existe una controversia acerca de si la aceptación de la vacuna disminuyó por cuenta del
ESAVI o si influyeron otros aspectos sociales no explorados en profundidad. Por tanto, a partir del marco conceptual de los determinantes sociales de la salud (DSS), como las "condiciones de vida, de trabajo, de acceso a la salud, aspectos culturales, de educación en un contexto particular" (17), el objetivo de esta revisión fue determinar si aspectos sociales como educación (de padres, niños, adolescentes y profesionales de salud); acceso a la vacuna de VPH (costos, nivel socioeconómico, región); cobertura de medios de comunicación y la relación con el personal de salud podrían haber afectado la aceptación de la vacuna contra el VHP en mujeres colombianas, más allá del evento de Carmen de Bolívar, y sintetizar la información de acuerdo con los aspectos mencionados.

\section{MATERIALES Y MÉTODOS}

La pregunta por responder en esta investigación es: ¿qué aspectos sociales podrían estar asociados a la reducción de las tasas de vacunación contra el VPH en Colombia? Para responderla se realizó una búsqueda sistemática de literatura publicada en español e inglés en 6 bases de datos indexadas (Scopus, Web of Science, Medline vía PubMed, Embase, Biblioteca Virtual en Salud y Ovid), a partir de los términos de búsqueda especificados en el Anexo 1. Debido al interés por conocer la aceptación de la vacunación contra el VPH desde una perspectiva social, se incluyó un amplio espectro de documentos de la literatura gris, la cual se consultó en Google Scholar, repositorios de la Universidad Nacional de Colombia, Universidad de Antioquia, Universidad del Valle, Pontificia Universidad Javeriana y Universidad del Rosario, así como páginas web de entidades como el Ministerio de Salud y Protección Social (MSPS), Instituto Nacional de Salud (INS), Instituto de Evaluación y Tecnología de Salud (IETS), Instituto Nacional de Cancerología (INC) e Instituto Nacional de Vigilancia de Medicamentos y Alimentos (Invima), en el periodo comprendido entre enero de 2006 y abril de 2018. 
Criterios de inclusión por tipo de documentos: se buscaron revisiones sistemáticas, ensayos clínicos controlados, estudios observacionales, estudios cualitativos y revisiones narrativas que tuvieran en cuenta aspectos sociales que influenciaron la aceptación de la vacunación contra el VPH en la población femenina colombiana susceptible de ser vacunada. También se tuvieron en cuenta otros documentos tales como editoriales, notas al editor y circulares gubernamentales que tuvieran información que diera respuesta a la pregunta de investigación propuesta. Se realizó tamizaje de la literatura basado en título y resumen, utilizando el software Covidence (18); la selección final se efectuó después de la lectura completa del texto de manera independiente por dos revisores (FP y SJEC); las diferencias se resolvieron por consenso.

De manera independiente se evaluó la calidad del reporte de los documentos incluidos, empleando las guías recomendadas por la red EQUATOR (19) para los diferentes tipos de estudio en epidemiología y salud pública (STROBE, COREQ y CHEERS) (20-22); así como las guías de lectura crítica de Joanna Briggs para texto de opinión (23). Para los artículos de revisión narrativa se utilizó el instrumento propuesto por Guirao Goris et al. (24). En caso de una revisión narrativa más estructurada metodológicamente se usó la guía de lectura crítica de CASPe (25).

Los resultados se presentan de manera narrativa y se organizaron de acuerdo con las categorías definidas a priori y por consenso entre los autores sobre los aspectos sociales basados en el marco de los DSS: educación, acceso a la vacuna, rol de los medios de comunicación y relación con el personal de salud, que pudieron afectar la aceptación de la vacuna por parte de la población. La síntesis de las características de los documentos seleccionados incluyó: autor, diseño, calidad de la evidencia y aspectos sociales que condicionaron la aceptación (tabla 1).

El protocolo de esta revisión fue registrado en International Prospective Register of Systematic Reviews (Prospero), bajo el código CRD42018100755.

\section{RESULTADOS}

Se identificó un total de 3.708 registros, de los cuales 129 fueron elegidos para evaluar texto completo; finalmente, se seleccionaron 24 para síntesis cualitativa (figura 1).

Características generales de los estudios: En cuanto al diseño, 8 documentos de tipo cualitativo (33,3\%) los cuales se distribuyeron según su diseño (26) en tres tipos: 3 interpretativos (27-29), 1 mixto (30) y 4 narrativos (31-34). Un total de 9 correspondieron a estudios de tipo cuantitativo (37,5\%): 2 estudios de caso $(35,36), 1$ evaluación económica (37), 6 estudios de corte transversal (15,16,38-41); 3 documentos (12,5\%) correspondieron a revisiones narrativas (42-44) y 4 (45-48) a otros (16,7\%).

Calidad del reporte de la publicación: en general, la evaluación de la calidad del reporte de los documentos incluidos en las listas de chequeo mostró porcentajes de cumplimiento mayores al $80 \%$ en 6 documentos $(27,28,37,46-48), 17$ en un rango intermedio, 60-79\% (15,16,29-32,34-36,38-45) 1 en un rango bajo, $<60 \%$ (33).

\section{Aspectos sociales evaluados}

Educación: un total de 19 (79\%) estudios evaluó aspectos educativos en grupos de padres, adolescentes, profesionales de la salud y tomadores de decisiones, que han influenciado la vacunación contra VPH en Colombia (15,27,29-35,38,39,41-46,48). Respecto a los padres, los aspectos educativos no son percibidos como orientados hacia la prevención del CCU, sino hacia la prevención de una infección de transmisión sexual (ITS). Existen creencias en los padres de que la vacunación inducirá el inicio temprano de relaciones sexuales en sus hijas y que las vacunas solo son para población menor de 5 años de edad (28). Hay evidencia que muestra que después de recibir información adecuada sobre los beneficios de la vacunación, los padres entendían la importancia de vacunar a sus hijas y accedían a hacerlo $(34,41)$. Un total de 5 documentos se enfocan en educación en adolescentes $(15,31,34,41,45)$, 


\begin{tabular}{|c|c|c|c|c|}
\hline Autor y año & $\begin{array}{l}\text { Tipo de } \\
\text { documento }\end{array}$ & $\begin{array}{c}\text { Grupos o fuentes } \\
\text { de información sobre } \\
\text { las cuales se hace el } \\
\text { análisis }\end{array}$ & $\begin{array}{l}\text { Aspectos sociales } \\
\text { que afectaron la } \\
\text { aceptación de la } \\
\text { vacunación }\end{array}$ & $\begin{array}{l}\text { Calidad de la evidencia } \\
\text { (\% cumplimiento de la } \\
\text { lista de chequeo } \\
\text { empleada para su } \\
\text { evaluación) }\end{array}$ \\
\hline Anaya (35) & $\begin{array}{l}\text { Estudio } \\
\text { de casos }\end{array}$ & $\begin{array}{l}3 \text { mujeres vacunadas } \\
\text { contra el VPH }\end{array}$ & Educación & $63 \%-C A R E$ \\
\hline $\begin{array}{l}\text { Aponte- } \\
\text { González (37) }\end{array}$ & $\begin{array}{l}\text { Evaluación } \\
\text { económica }\end{array}$ & $\begin{array}{l}\text { Cohorte hipotética de } \\
\text { mujeres de } 12 \text { a } 76 \text { años }\end{array}$ & Acceso a la vacuna & $87,5 \%$-CHEERS \\
\hline Benavides(27) & Ensayo & Padres de familia & $\begin{array}{l}\text { Educación, relación } \\
\text { personal de salud }\end{array}$ & $\begin{array}{l}83 \%-J \text { Briggs Institute Opi- } \\
\text { nion Paper Text }\end{array}$ \\
\hline $\begin{array}{l}\text { Bermedo- } \\
\text { Carrasco (39) }\end{array}$ & $\begin{array}{l}\text { Estudio de } \\
\text { corte transversal }\end{array}$ & $\begin{array}{l}53521 \text { mujeres } 13-49 \\
\text { años }\end{array}$ & Educación & $77,3 \%$-STROBE \\
\hline $\begin{array}{l}\text { Bermedo- } \\
\text { Carrasco (38) }\end{array}$ & $\begin{array}{l}\text { Estudio de } \\
\text { corte transversal }\end{array}$ & $\begin{array}{l}53521 \text { mujeres 13-49 } \\
\text { años }\end{array}$ & $\begin{array}{l}\text { Educación, acceso a } \\
\text { la vacuna }\end{array}$ & $68,2 \%-S T R O B E$ \\
\hline $\begin{array}{l}\text { Caro Martínez } \\
\text { (36) }\end{array}$ & Estudio de caso & $\begin{array}{l}\text { Entes evaluadores de tec- } \\
\text { nologías en salud de tres } \\
\text { países: Colombia, Brasil } \\
\text { y Polonia }\end{array}$ & Acceso a la vacuna & 66,6\%-COREQ \\
\hline $\begin{array}{l}\text { Cortes-García, } \\
(30)\end{array}$ & $\begin{array}{l}\text { Tesis de doc- } \\
\text { torado salud } \\
\text { pública estudio } \\
\text { basado en varias } \\
\text { metodologías } \\
\text { cualitativas }\end{array}$ & $\begin{array}{l}\text { Mujeres wayuu (20-45 } \\
\text { años), residentes en área } \\
\text { urbana y rural. } \\
\text { Funcionarios de salud } \\
\text { de nivel local y departa- } \\
\text { mental (tomadores de } \\
\text { decisión y prestadores de } \\
\text { salud) }\end{array}$ & $\begin{array}{l}\text { Educación, relación } \\
\text { con el personal de } \\
\text { salud, cubrimiento } \\
\text { de medios de comu- } \\
\text { nicación }\end{array}$ & $66.1 \%-C O R E Q$ \\
\hline $\begin{array}{l}\text { Téllez-Pedroza, } \\
\text { (31) }\end{array}$ & $\begin{array}{l}\text { Tesis de docto- } \\
\text { rado en ciencias } \\
\text { sociales, estudio } \\
\text { cualitativo ba- } \\
\text { sado en grupos } \\
\text { focales }\end{array}$ & $\begin{array}{l}\text { Niñas, padres de familia, } \\
\text { profesores, prestadores } \\
\text { de servicios de salud re- } \\
\text { lacionados con el evento } \\
\text { masivo de Carmen de } \\
\text { Bolívar }\end{array}$ & $\begin{array}{l}\text { Educación, relación } \\
\text { con el personal de } \\
\text { salud, cubrimiento } \\
\text { de medios de comu- } \\
\text { nicación }\end{array}$ & $66 \%-C O R E Q$ \\
\hline $\begin{array}{l}\text { Jiménez- } \\
\text { Cendales (16) }\end{array}$ & $\begin{array}{l}\text { Trabajo de } \\
\text { grado de espe- } \\
\text { cialización en } \\
\text { epidemiología, } \\
\text { estudio de corte } \\
\text { transversal }\end{array}$ & $\begin{array}{l}\text { Base de datos del Progra- } \\
\text { ma Ampliado de Inmu- } \\
\text { nización 2011-2014 }\end{array}$ & $\begin{array}{l}\text { Cubrimiento de } \\
\text { medios de comuni- } \\
\text { cación }\end{array}$ & 72,5\%-STROBE \\
\hline $\begin{array}{l}\text { Maldonado } \\
(28)\end{array}$ & Ensayo & $\begin{array}{l}\text { Proceso de introducción } \\
\text { de la vacuna contra el } \\
\text { VPH en la población de } \\
\text { Colombia }\end{array}$ & $\begin{array}{l}\text { Relación con el per- } \\
\text { sonal de salud, cu- } \\
\text { brimiento de medios } \\
\text { de comunicación }\end{array}$ & $\begin{array}{l}100 \% \text {-J Briggs Institute } \\
\text { Opinion Paper Text }\end{array}$ \\
\hline
\end{tabular}




\begin{tabular}{|c|c|c|c|c|}
\hline Autor y año & $\begin{array}{l}\text { Tipo de } \\
\text { documento }\end{array}$ & $\begin{array}{c}\text { Grupos o fuentes } \\
\text { de información sobre } \\
\text { las cuales se hace el } \\
\text { análisis }\end{array}$ & $\begin{array}{l}\text { Aspectos sociales } \\
\text { que afectaron la } \\
\text { aceptación de la } \\
\text { vacunación }\end{array}$ & $\begin{array}{l}\text { Calidad de la evidencia } \\
\text { (\% cumplimiento de la } \\
\text { lista de chequeo } \\
\text { empleada para su } \\
\text { evaluación) }\end{array}$ \\
\hline Martínez (15) & $\begin{array}{l}\text { Informe } \\
\text { epidemiológico, } \\
\text { estudio de corte } \\
\text { transversal }\end{array}$ & $\begin{array}{l}\text { Niños, adolescentes y } \\
\text { adultos jóvenes residen- } \\
\text { tes en el municipio de El } \\
\text { Carmen de Bolívar. que } \\
\text { hayan consultado entre } \\
\text { el } 28 \text { de mayo y el } 15 \text { de } \\
\text { octubre de } 2014\end{array}$ & $\begin{array}{l}\text { Educación, relación } \\
\text { con el personal de } \\
\text { salud }\end{array}$ & 77,3\%-STROBE \\
\hline MSPS (45) & $\begin{array}{l}\text { Circular } \\
\text { gubernamental }\end{array}$ & $\begin{array}{l}\text { Circular } 0062 \text { de } 2014 . \\
\text { Vacunación contra el } \\
\text { VPH en niñas de cuarto } \\
\text { grado de básica primaria } \\
\text { de } 9 \text { años y más de edad, } \\
\text { y niñas desescolarizadas } \\
\text { de nueve a diecisiete } \\
\text { años en el territorio } \\
\text { nacional. }\end{array}$ & $\begin{array}{l}\text { Educación, relación } \\
\text { con el personal de } \\
\text { salud }\end{array}$ & $\begin{array}{l}66,6 \%-\text { Briggs Institute } \\
\text { Opinion Paper Text }\end{array}$ \\
\hline $\begin{array}{l}\text { Molina Sierra } \\
(29)\end{array}$ & $\begin{array}{l}\text { Trabajo de } \\
\text { grado de } \\
\text { especialización } \\
\text { en bioética. } \\
\text { Ensayo }\end{array}$ & $\begin{array}{l}\text { Implicaciones bioéticas } \\
\text { de la introducción de la } \\
\text { vacuna contra el virus } \\
\text { del papiloma humano } \\
\text { en niñas colombianas } \\
\text { menores de } 14 \text { años }\end{array}$ & Educación & $\begin{array}{l}66,6 \% \text { - Briggs Institute } \\
\text { Opinion Paper Text }\end{array}$ \\
\hline $\begin{array}{l}\text { Nogueira- } \\
\text { Rodrigues (46) }\end{array}$ & Comentario & $\begin{array}{l}\text { Programas de vacunación } \\
\text { de Latinoamérica }\end{array}$ & $\begin{array}{l}\text { Educación, Acceso } \\
\text { a la vacuna }\end{array}$ & $\begin{array}{l}83,3 \% \text { - Briggs Institute } \\
\text { Opinion Paper Text }\end{array}$ \\
\hline Nwanodi (42) & $\begin{array}{l}\text { Revisión } \\
\text { narrativa }\end{array}$ & $\begin{array}{l}45 \text { artículos sobre acti- } \\
\text { tudes hacia la vacunación } \\
\text { en la región andina }\end{array}$ & $\begin{array}{l}\text { Educación, cu- } \\
\text { brimiento de los } \\
\text { medios de comuni- } \\
\text { cación }\end{array}$ & $\begin{array}{l}73,3 \text { \%- Guías CASPe de } \\
\text { Lectura Critica }\end{array}$ \\
\hline Nwanodi (43) & $\begin{array}{l}\text { Revisión narra- } \\
\text { tiva }\end{array}$ & $\begin{array}{l}36 \text { artículos incluidos so- } \\
\text { bre la vacunación contra } \\
\text { el VPH en región andina }\end{array}$ & $\begin{array}{l}\text { Educación, } \\
\text { cubrimiento de los } \\
\text { medios de comuni- } \\
\text { cación }\end{array}$ & $\begin{array}{l}\text { 73,3 \%- Guías CASPe de } \\
\text { Lectura Critica }\end{array}$ \\
\hline Piñeros (32) & $\begin{array}{l}\text { Estudio cuali- } \\
\text { tativo con base } \\
\text { en entrevistas } \\
\text { semiestructu- } \\
\text { radas }\end{array}$ & $\begin{array}{l}\text { Secretario general de } \\
\text { salud, el jefe de salud } \\
\text { pública del departamen- } \\
\text { to, profesionales respon- } \\
\text { sables de salud sexual y } \\
\text { reproductiva y progra- } \\
\text { mas de inmunización }\end{array}$ & $\begin{array}{l}\text { Educación, relación } \\
\text { con el personal de } \\
\text { salud }\end{array}$ & 66,6\%- COREQ \\
\hline Piñeros (33) & $\begin{array}{l}\text { Estudio cualita- } \\
\text { tivo con base en } \\
\text { grupos focales }\end{array}$ & $\begin{array}{l}\text { Médicos generales, pe- } \\
\text { diatras y ginecólogos }\end{array}$ & $\begin{array}{l}\text { Educación, relación } \\
\text { con el personal de } \\
\text { salud }\end{array}$ & 55,5\%- COREQ \\
\hline Piñeros (40) & $\begin{array}{l}\text { Estudio de corte } \\
\text { transversal }\end{array}$ & $\begin{array}{l}\text { Hombres y mujeres } \\
\text { entre 18-45 años con } \\
\text { lesiones sospechosas de } \\
\text { verrugas genitales. }\end{array}$ & $\begin{array}{l}\text { Educación, relación } \\
\text { con el personal de } \\
\text { salud }\end{array}$ & 77,3\%-STROBE \\
\hline
\end{tabular}




\begin{tabular}{|c|c|c|c|c|}
\hline Autor y año & $\begin{array}{c}\text { Tipo de } \\
\text { documento }\end{array}$ & $\begin{array}{c}\text { Grupos o fuentes } \\
\text { de información sobre } \\
\text { las cuales se hace el } \\
\text { análisis }\end{array}$ & $\begin{array}{l}\text { Aspectos sociales } \\
\text { que afectaron la } \\
\text { aceptación de la } \\
\text { vacunación }\end{array}$ & $\begin{array}{l}\text { Calidad de la evidencia } \\
\text { (\% cumplimiento de la } \\
\text { lista de chequeo } \\
\text { empleada para su } \\
\text { evaluación) }\end{array}$ \\
\hline $\begin{array}{l}\text { Sánchez- } \\
\text { Gómez (47) }\end{array}$ & Editorial & $\begin{array}{l}\text { Programa de vacuna- } \\
\text { ción contra el VPH en } \\
\text { Colombia }\end{array}$ & $\begin{array}{l}\text { Educación, relación } \\
\text { con el personal de } \\
\text { salud }\end{array}$ & $\begin{array}{l}\text { 83,3\%-Briggs Institute } \\
\text { Opinion Paper Text }\end{array}$ \\
\hline Reina (48) & Editorial & $\begin{array}{l}\text { Programa de vacuna- } \\
\text { ción contra el VPH en } \\
\text { Colombia }\end{array}$ & $\begin{array}{l}\text { Educación, relación } \\
\text { con el personal de } \\
\text { salud }\end{array}$ & $\begin{array}{l}100 \%-\text { Briggs Institute } \\
\text { Opinion Paper Text }\end{array}$ \\
\hline $\begin{array}{l}\text { Salazar-Fajardo } \\
(44)\end{array}$ & $\begin{array}{l}\text { Revisión narra- } \\
\text { tiva }\end{array}$ & $\begin{array}{l}97 \text { documentos sobre la } \\
\text { estrategia de vacunación } \\
\text { contra VPH }\end{array}$ & $\begin{array}{l}\text { Educación, relación } \\
\text { con el personal de } \\
\text { salud, cubrimiento } \\
\text { de medios de comu- } \\
\text { nicación }\end{array}$ & $\begin{array}{l}66,6 \% \text { - Guías CASPe de } \\
\text { Lectura Crítica }\end{array}$ \\
\hline $\begin{array}{l}\text { Torrado- } \\
\text { Arenas (41) }\end{array}$ & $\begin{array}{l}\text { Estudio de corte } \\
\text { transversal }\end{array}$ & $\begin{array}{l}\text { Padres con hijos/hijas } \\
<18 \text { años }\end{array}$ & $\begin{array}{l}\text { Educación, relación } \\
\text { con el personal de } \\
\text { salud }\end{array}$ & $63,6 \%-S T R O B E$ \\
\hline Wiesner (34) & $\begin{array}{l}\text { Estudio cualita- } \\
\text { tivo con base en } \\
\text { grupos focales }\end{array}$ & $\begin{array}{l}\text { Padres de adolescentes } \\
\text { entre } 11 \text { y } 14 \text { años de } \\
\text { estudiantes de colegios } \\
\text { públicos y privados }\end{array}$ & $\begin{array}{l}\text { Acceso a la vacuna, } \\
\text { educación }\end{array}$ & 77,7 \%-COREQ \\
\hline
\end{tabular}

Fuente: elaboración propia.

uno de ellos señala un bajo conocimiento de la existencia de la vacuna en esta población debido a la baja frecuencia en solicitud de consulta médica y a que son los padres quienes toman la decisión (31). Otros 8 estudios se enfocaron en educación en profesionales de la salud $(15,27,30-33,44,45)$; uno de estos indica que no hay conocimiento adecuado en médicos generales, personal de salud y tomadores de decisión sobre los eventos adversos y cómo entregar la información de estos (33). Respecto a los tomadores de decisiones, los funcionarios encargados de programas de salud en la comunidad no cuentan con la suficiente información sobre este asunto (32). De igual manera, en una revisión narrativa se señala que es necesario articular los ámbitos de salud y educación en la política pública (44). En general, se evidenciaron aspectos como: la preocupación por parte de médicos y padres de familia de que las farmacéuticas sean quienes asuman la educación relacionada con la vacunación $(33,41)$.
Acceso a la vacuna: este aspecto fue analizado en 7 estudios $(29,1 \%)(29,34,36-39,46)$. Desde esta perspectiva, una barrera era el costo de la vacuna antes de ser introducida en el PAI (34), otra barrera era vivir en zonas rurales $(38,39)$. En Colombia, Aponte et al., en 2013, encontraron que la vacuna no era costo-efectiva, por el precio, desde la perspectiva social (37). De hecho, Piñeros encontró que para 2013, el alto costo de la vacuna impedía su accesibilidad, aunque algunos padres estarían dispuestos a asumirlos por el beneficio que podía llegar a representar para sus hijas. En general, los tomadores de decisiones coincidieron en que el costo de la vacuna puede afectar su aceptación, y que esto puede retrasar su masificación (32). Por tal razón, este programa de vacunación debería ser cubierto por el sistema de salud para incrementar su aceptación en la región latinoamericana (44). Un estudio de caso realizado después de la inclusión en el PAI con entes de evaluación de tecnología de 
Figura 1.

Diagrama PRISMA, proceso de selección de artículos.

Número de registros identificados (Scopus: 45 -Web of Science: 214

-PubMed: 54 -Embase: 291 -BVS:33

-Ovid: 17) $(\mathrm{n}=654)$
Número de registros identificados en otras fuentes (Google académico: 2834 -Repositorios universidades colombianas: 707 -Páginas web de entes gubernamentales de salud: 56)

$$
(\mathrm{n}=3.597)
$$

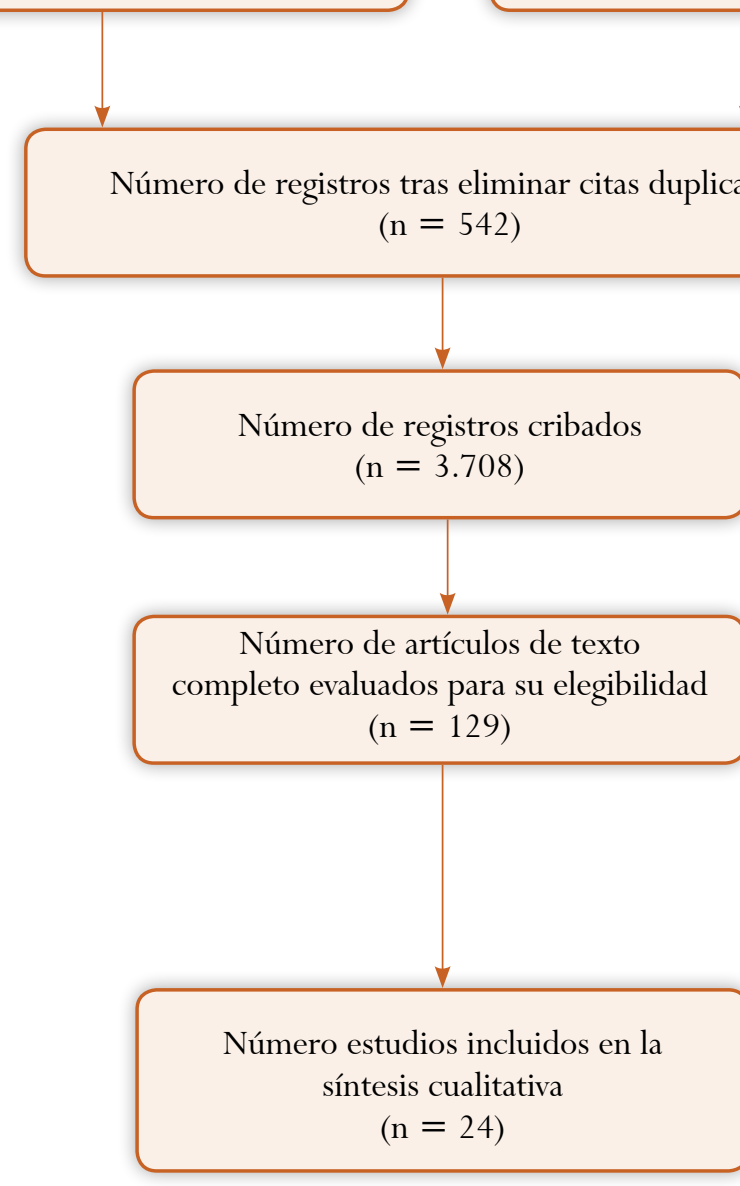

Número de registros excluidos al no cumplir criterios de inclusión en la fase de cribado $(n=3.579)$

Número de artículos de texto completo excluidos, con sus razones

$$
(n=104)
$$

55 no corresponden al objeto de investigación

27 no incluyen aspectos sociales que impacten tasas de vacunación 16 no incluyen la población a estudio 4 no dentro periodo de tiempo de la revisión 3 no mencionan la vacuna tetravalente

Fuente: elaboración propia.

salud de tres países, entre ellos Colombia encontró que el acceso a la vacuna del VPH podría tener dificultades debido a cuestiones burocráticas y cambios institucionales (36).

Relación con el personal de salud: este aspecto fue abordado en 8 estudios (33\%) (27,28,31$33,41,44,45)$. Cuatro estudios señalan que el personal de salud frecuentemente no escucha las inquietudes relacionadas con la vacuna en las poblaciones de escasos recursos $(31,34,41,46)$. Por otra parte, otros estudios informan que la falta de suficiente información relacionada con eventos adversos, por parte de los profesionales de la salud, puede generar ansiedad en los padres $(27,28,32,41)$.Por último, que la información se entrega sin tener en cuenta las particularidades de quien la recibe (36). Se ha sugerido que el personal de salud debe adoptar el rol de educador frente a la comunidad sobre la seguridad 
de la vacuna a fin de evitar asociaciones erróneas de esta con eventos adversos (45).

Los medios de comunicación: un total de 8 estudios (33\%) tienen en cuenta este aspecto, que es de suma importancia para implementar los programas de vacunación $(15,16,28,31,42-44,47)$. Un estudio describe que la cobertura de los medios sobre los eventos adversos, así no sean de la magnitud de lo sucedido en el Carmen de Bolívar, impacta la aceptación de la vacuna por parte de la comunidad (31). De otra parte, la información emitida por los medios de comunicación puede no solamente confundir a la opinión pública, sino a miembros de la comunidad científica (16). Otro asunto relacionado con la cobertura de los medios es el hecho de crear tensiones entre los padres y los expertos al momento de informar con relación a las vacunas, en particular eventos adversos (28). No obstante lo anterior, la cobertura de los medios también tiene una gran influencia para facilitar la aceptación de programas de vacunación $(27,28,42,43,47)$.

\section{DISCUSIÓN}

Esta revisión de la literatura evidencia que las tasas y la cobertura de vacunación alcanzadas disminuyeron por aspectos sociales como la educación, la relación con el personal de salud y el manejo mediático dado, así como por aspectos relacionados con el acceso a la vacuna en Colombia, además de los ESAVI en el Carmen de Bolívar en 2014 $(16,27,28,44,46,47)$.

Respecto a la educación, se identificó la importancia del conocimiento del VPH y su relación con el CCU. En cuanto a los profesionales de la salud, se identifica la relevancia de una buena comunicación para informar adecuadamente tanto los beneficios como los potenciales eventos adversos de la vacuna pues cuentan con el conocimiento y, en algunas comunidades, con la credibilidad en su papel de expertos (40). El personal sanitario deberá mejorar la comunicación sobre seguridad de la vacuna y fortalecer el vínculo con los adolescentes y sus familias
(27). La inclusión de una vacuna en el PAI es una intervención que facilita el acceso a esta por parte de la población, sin importar su nivel de ingreso (36).

Ante ello, es necesario tener estrategias como la atención centrada en el paciente que busquen persuadir y, por tanto, aceptar vacunarse más que obligar, lo que implica el fortalecimiento de la relación médico-paciente para que el personal de salud sea una fuente de información creíble y legítima $(27,39)$.

A partir de los resultados, se considera que existe la necesidad de implementar programas educativos para la población general acerca del CCU y su relación con el VPH con el objeto de aumentar el conocimiento acerca de esta asociación y favorecer la vacunación (39). De otra parte, con respecto a las campañas de vacunación, estas han evitado focalizarse en la cuestión de la sexualidad, pese a que la literatura sugiere el importante papel que juega la comprensión de la transmisión sexual del VPH y su relación con la justificación de la vacunación; lo anterior, probablemente para evitar la asociación de la vacuna con un inicio temprano de las relaciones sexuales, impidiendo la aceptación por los padres (28).

Es necesario tener presente que los programas nacionales de vacunación, al ser intervenciones de política pública en salud, tienen una dimensión social intrínseca y deben considerar estos aspectos de la población donde se implementa, no solamente para su éxito, sino para lograr el impacto deseado. Por tanto, es pertinente educar a la comunidad adecuadamente sobre los beneficios y riesgos de la vacunación, porque si no se tiene certeza acerca de la seguridad de la vacunación, esto desfavorecerá su aceptación $(34,41)$.

Se necesita instruir a los comunicadores sociales sobre temas de salud para que orienten adecuadamente a la opinión pública (15). Asimismo, la comunicación en intervenciones de este tipo debería ser liderada por la autoridad en salud y no por otras fuentes $(40,41)$, porque se puede distorsionar el mensaje. 
Se requiere tener una comunicación directa y transparente con la comunidad frente a posibles eventos adversos, además del manejo coordinado de los canales de comunicación institucionales con los medios, dado que la promoción de la vacuna se realizó a través de televisión, radio y prensa (31), para que los medios sean un aliado al informar sobre estas intervenciones.

Es importante que la información que reciban las jóvenes y los padres de familia sea válida, confiable y ajustada a las características socioeconómicas y educativas de la población a la cual va a ser entregada $(15,31,33,41)$. Por otra parte, los eventos adversos de la vacuna presentados en las niñas y adolescentes de Carmen de Bolívar pusieron de manifiesto la necesidad de que la autoridad sanitaria tenga mayor credibilidad. Para alcanzarla se sugiere que tengan más información relacionada con farmacovigilancia $(31,36)$, basada en un efectivo seguimiento de los eventos adversos en la población vacunada, puesto que pueden presentarse fallas en su procesos de notificación y clasificación (16).

Para finalizar, en relación con los resultados, el tema del acceso a la vacuna se ha resuelto con el ingreso de esta al PAI, lo que hace que este asunto disminuya su importancia en la ponderación de aspectos sociales que influyen en la aceptación de la vacuna por parte de la población $(36,37)$.

La principal fortaleza de este estudio es la amplitud en la búsqueda de la literatura, si se compara con trabajos similares como los de Nwanodi, en relación con la vacunación contra el VPH $(42,43)$; este se limita a revisar PubMed, mientras que en esta investigación se realizó una búsqueda más amplia que abarcó también la literatura gris. De otra parte, la revisión de la literatura gris no solamente se limitó a Google Académico, sino que implicó la búsqueda sistemática en repositorios de las más reconocidas universidades en Colombia. Además, al tener en cuenta documentos con diversas metodologías (cualitativas-cuantitativas), se pudo desarrollar una revisión con una amplia perspectiva.
Dentro de las limitaciones se tienen las siguientes: el estudio tenía como objetivo mirar el problema en el contexto colombiano, lo que puede ser relativo porque si bien es cierto que le quita validez externa a las recomendaciones que pueden surgir de esta investigación, se tiene una alta validez interna con respecto a las recomendaciones que resulten de este trabajo en el contexto colombiano. Otra limitación, quizá, fue el no haber enfocado la búsqueda en documentos con una metodología exclusivamente cualitativa ante el temor de perder información que respondiera a la pregunta que dio a lugar a esta investigación, en documentos de tipo cuantitativo.

\section{CONCLUSIONES}

Es importante reconocer las implicaciones que para la práctica tienen los aspectos socioculturales de la población a fin de aumentar la aceptación de la vacuna, fortaleciendo y reforzando estrategias de educación para todos los interesados. Con relación a la comunicación del programa de vacunación del VPH es necesario involucrar y coordinar adecuadamente diferentes actores, como lo son: medios de comunicación, profesionales de la salud, e incluso a los docentes de los colegios. Es conveniente entrenar a los profesionales de la salud en un modelo de atención centrada en el paciente, con el objeto de que consoliden una mejor relación con estos. Hay que investigar más sobre las problemáticas alrededor de la infección por VPH y la vacuna contra este en la población y la manera como se entrega la información relacionada; además, se puede fortalecer la relación con el personal de salud, pues esto mejoraría la aceptación de esta medida de prevención.

\section{AGRADECIMIENTOS}

A la Liga Colombiana Contra el Cáncer por habernos invitado a participar en el proyecto: "Por la salud de la mujer araucana", el cual fue el punto de partida para desarrollar este trabajo. 


\section{FINANCIACIÓN}

Este proyecto no tuvo fuentes de financiación.

\section{REFERENCIAS}

1. World Health Organization. Human papillomavirus vaccines: WHO position paper. Wkly Epidemiol Rec. 2017;92(19):241-68.

2. Bosch FX, Lorincz A, Muñoz N, Meijer CJLM, Shah $\mathrm{KV}$. The causal relation between human papillomavirus and cervical cancer. J Clin Pathol. 2002;55(4):244-65. https://doi.org/10.1136/jcp.55.4.244

3. De Martel C, Ferlay J, Franceschi S, Vignat J, Bray F, Forman D, et al. Global burden of cancers attributable to infections in 2008: A review and synthetic analysis. Lancet Oncol. 2012;13(6):607-15. http:// doi.org/10.1016/S1470-2045(12)70137-7

4. WHO. Human papillomavirus (HPV). 2018. Disponible en: http://www.who.int/immunization/diseases/hpv/en/

5. IARC-WHO. Cervix uteri. Cancer incidence and mortality statistics worldwide and by region. 2018 . Disponible en: https://gco.iarc.fr/today/data/factsheets/cancers/23-Cervix-uteri-fact-sheet.pdf

6. Prat J, Franceschi S, Denny L, Lazcano E. Cancers of the female reproductive organs. World Cancer Report. IARC; 2014.

7. Stewart BW, Wild CP, editores. World cancer report 2014. Geneva: World Health Organization; 2014. Disponible en: https://publications.iarc.fr/Non-SeriesPublications/World-Cancer-Reports/World-CancerReport-2014

8. Bravo LE, Collazos T, Collazos P, García LS, Correa $\mathrm{P}$. Trends of cancer incidence and mortality in Cali, Colombia. 50 years experience. Colomb Med (Cali). 2012;1:246-55. https://doi.org/10.25100/ cm.v43i4.1266

9. Universidad del Valle. Facultad de Salud. Registro Poblacional de cáncer de Cali. Disponible en: http:// rpcc.univalle.edu.co/es/index.php

10. WHO. Immunization, Vaccines and Biologicals database. 2017. Disponible en: http://www.who.int/entity/ immunization/monitoring_surveillance/VaccineIntroStatus.pptx
11. Ministerio de Salud y Protección Social, Instituto Nacional de Cancerología, Instituto Nacional de Salud. Vacunación contra el virus papiloma humano - VPH en Colombia, para la prevención del cáncer de cuello uterino y verrugas genitales. 2014. Disponible en: https://www.minsalud.gov.co/sites/rid/Lists/BibliotecaDigital/RIDE/IA/INCA/1-vacunacion-contravirus-papilomahumano-verrugas-genitales.pdf

12. Congreso de Colombia. Ley 1626 del 30 de abril de 2013. "Por medio de la cual se garantiza la vacunación gratuita y obligatoria a la población colombiana objeto de la misma, se adoptan medidas integrales para la prevención del cáncer cérvico uterino y se dictan otras disposiciones. Gobierno de Colombia; 2013. Disponible en: https://www.minsalud.gov.co/sites/rid/ Lists/BibliotecaDigital/RIDE/INEC/IGUB/ley-1626de-2013.pdf

13. Ministerio de Salud y Protección Social. Colombia cuenta con las mejores coberturas de vacunación contra VPH del mundo. 2014. Disponible en: https://www. minsalud.gov.co/Paginas/Colombia-cuenta-con-lasmejores-coberturas-de-vacunacion.aspx

14. Ministerio de Salud y Protección Social / Grupo de Inmunoprevenibles. Jornada de Vacunación contra el Virus del Papiloma Humano. 2014. Disponible en: https://www.minsalud.gov.co/sites/rid/Lists/ BibliotecaDigital/RIDE/VS/PP/PAI/JORNADADEVACUNACIÓNCONTRAELVIRUSDELPAPILOMA HUMANO.pdf

15. Martínez M, Estévez A, Quijada H, Walteros D, Tolosa N, Paredes A, et al. Brote de evento de etiología desconocida en el municipio de El Carmen de Bolívar, Bolívar, 2014. Iqen. Informe quincenal epidemiológico. 2015;20(3-4): 41-76. Disponible en: https:// www.ins.gov.co/buscador-eventos/IQEN/IQEN\%20 vol\%2020\%202015\%20num\%203_4.pdf

16. Jiménez-Cendales BS. Descripción de eventos adversos relacionados con aplicación de vacuna contra el virus de papiloma humano en Bogotá. Universidad del Rosario; 2015. Disponible en: http://repository. urosario.edu.co/handle/10336/11322

17. Marmot M, Allen J. Health priorities and the social determinants of health. East Mediterr Heal J. 2015;21(9):6712. https://doi.org/10.26719/2015.21.9.671 
18. Veritas Health Innovation. Covidence systematic review software Melbourne; 2020. Disponible en: https://www.covidence.org

19. The EQUATOR Network. Enhancing the QUAlity and Transparency of Health Research. Reporting guidelines for main study types. 2014. Disponible en: http://www. equator-network.org/

20. von Elm E, Altman DG, Egger M, Pocock SJ, Gøtzsche PC, Vandenbroucke JP, et al. The strengthening the reporting of observational studies in epidemiology (STROBE) statement: Guidelines for reporting observational studies. Ann Intern Med. 2007;147(8):573-7. https://doi.org/10.7326/00034819-147-8-200710160-00010

21. Tong A, Sainsbury P, Craig J. Consolidated criteria for reporting qualitative research (COREQ): A 32item checklist for interviews and focus group. Int J Qual Health Care. 2007;19(6):349-57. https://doi. org/10.1093/intqhc/mzm042

22. Husereau D, Drummond M, Petrou S, Carswell C, Moher D, Greenberg D, et al. Consolidated health economic evaluation reporting standards (CHEERS) statement. Value Health. 2013;16(2):e1-5. https:// doi.org/10.1016/j.jval.2013.02.010

23. The Joanna Briggs Institute. Checklist for text and opinion The Joanna Briggs Institute. Critical appraisal tools for use in JBI systematic reviews. Disponible en: https://joannabriggs.org/sites/default/files/2019-05/ JBI_Critical_Appraisal-Checklist_for_Text_and_ Opinion2017_0.pdf

24. Guirao-Goris J, Olmedo-Salas A, Ferrer Ferrandiz E. El artículo de revisión. Rev Iberoam Enferm Comunitaria. 2008;1(6):1-25. Disponible en: https://www. uv.es/joguigo/valencia/Recerca_files/el_articulo_de_ revision.pdf

25. Cabello JB. Programa de habilidades en lectura crítica español. Plantilla para ayudarte a entender una revisión sistemática. En: CASPe. Guías CASPe de lectura crítica de la literatura médica. Alicante: CASPe; 2005. p. 13-7. Disponible en: http://www.redcaspe.org/herramientas/instrumentos
26. Universidad de Jaén. Metodología Cualitativa. 2019. Disponible en: http://www.ujaen.es/investiga/tics_tfg/ enfo_cuali.html

27. Benavides M, Salazar L. Razones que pueden explicar la reducción en la cobertura de vacunación contra VPH en Colombia. Rev CES Salud Pública. 2017;8(1):82-93.

28. Maldonado OJ. Evidence, sex and state paternalism: Intersecting global connections in the introduction of HPV vaccines in Colombia BT. En: Johnson E, editor. Gendering Drugs: Feminist Studies of Pharmaceuticals. Springer International Publishing; 2017. p. 12958. https://doi.org/10.1007/978-3-319-51487-1_7

29. Molina Sierra OC. Implicaciones bioéticas de la introducción de la vacuna contra el virus del papiloma humano en niñas colombianas menores de 14 años [Especialización]. Universidad de La Sabana; 2017. Disponible en: http:/hdl.handle.net/10818/31142

30. Cortes-García C. Representaciones sociales del cáncer de cuello uterino en mujeres wayuu, en prestadores de servicios de salud y en tomadores de decisión del municipio de Uribia del departamento de La Guajira, Colombia [Doctorado]. Universidad Nacional de Colombia; 2016. Disponible en: http://bdigital.unal. edu.co/53097/1/35421080.2016.pdf

31. Téllez-Pedroza M. The controversy over the use of HPV vaccine in Colombia, centered on the "adverse vaccine reactions/mass hysteria" event in El Carmen de Bolívar, 2014 [Doctorado]. Universidad Nacional de Colombia; 2018. Disponible en: http://bdigital. unal.edu.co/64244/1/52966926.2018.pdf

32. Piñeros M, Wiesner C, Cortés C, Trujillo LM. HPV vaccine introduction at the local level in a developing country: Attitudes and criteria among key actors. Cad Saude Publica. 2010;26(5):900-8. https://doi. org/10.1590/S0102-311X2010000500012

33. Piñeros M, Cortés C, Trujillo L, Wiesner C. Conocimientos, aceptabilidad y actitudes sobre la vacuna contra el VPH en médicos generales, ginecólogos y pediatras en Colombia. Rev Colomb Cancerol. 2009;13(2):88-98. https://doi.org/10.1016/S01239015(09)70140-4 
34. Wiesner C, Piñeros M, Trujillo LM, Cortés C, Ardila J. Aceptabilidad de la vacuna contra el Virus Papiloma Humano en padres de adolescentes, en Colombia. Rev Salud Publica. 2010;12(6):961-73. Disponible en: http://www.scielosp.org/scielo.php? script $=$ sci_artte xt\&pid=S0124-00642010000600008

35. Anaya JM, Reyes B, Perdomo-Arciniegas AM, Camacho-Rodríguez B, Rojas-Villarraga A. Autoimmune/ autoinflammatory syndrome induced by adjuvants (ASIA) after quadrivalent human papillomavirus vaccination in Colombians: a call for personalised medicine. Clin Exp Rheumatol. 2015;33(4):545-8.

36. Caro Martínez A, Espín Balbino J, Lemgruber A, Martín Ruiz E, Olry de Labry Lima A, García-Mochón L, et al. Adoption of the HPV vaccine: A case study of three emerging countries. J Comp Eff Res. 2017;6(3):195-204. https://doi.org/10.2217/cer2016-0071

37. Aponte-González J, Fajardo-Bernal L, Diaz J, EslavaSchmalbach J, Gamboa O, Hay JW. Cost-effectiveness analysis of the bivalent and quadrivalent human papillomavirus vaccines from a societal perspective in Colombia. PLoS One. 2013;18;8(11):e80639. https:// doi.org/10.1371/journal.pone.0080639

38. Bermedo-Carrasco S, Waldner C, Peña-Sánchez JN, Szafron M. Spatial variations in cervical cancer prevention in Colombia: Geographical differences and associated socio-demographic factors. Spat Spatiotemporal Epidemiol. 2016;19:78-90. https://doi.org/10.1016/j. sste.2016.07.002

39. Bermedo-Carrasco S, Feng CX, Peña-Sánchez JN, Lepnurm R. Predictors of having heard about human papillomavirus vaccination: Critical aspects for cervical cancer prevention among Colombian women. Gac Sanit. 2015;29(2):112-7. https://doi.org/10.1016/j. gaceta.2014.09.005

40. Piñeros M, Hernández-Suárez G, Orjuela L, Vargas JC, Pérez G. HPV knowledge and impact of genital warts on self esteem and sexual life in Colombian patients. BMC Public Health. 2013;13(1). https:// doi.org/10.1186/1471-2458-13-272
41. Torrado-Arenas DM, Álvarez-Pabón Y, GonzálezCastañeda JL, Rivera-Contreras OE, Sosa-Vesga CD, González BX, et al. Conocimientos sobre el virus del papiloma humano y su vacuna en padres de familia de Rivera, Huila, en el 2015. Rev Médicas UIS. 2017;30(1):13-9. https://.doi.org/10.18273/revmed. v30n1-2017001

42. Nwanodi O. Attitudes towards human papilloma virus vaccination in the Latin American Andean Region. Healthcare. 2017;5(3):55. https://doi.org/10.3390/ healthcare 5030055

43. Nwanodi O. Eliminating andean region health disparities via human papilloma virus vaccination administration programs implementation. J Gynecol. 2017;2(3):000142.

44. Salazar Fajardo LJ, Benavides Delgado MR, Boogaard S, Marín Y. Estrategias latinoamericanas para la vacunación contra el virus del papiloma humano: una revisión temática. Hacia promoc Salud. 2017;22(2):12943. Disponible en: http://www.scielo.org.co/scielo. php?pid $=$ S0121-75772017000200129\&script $={ }_{\text {sci }}$ abstract\&tlng $=\mathrm{es}$

45. Ministerio de Salud y Protección Social. Circular 0062 de 2014. Vacunación contra el VPH en niñas de cuarto grado de básica primaria de nueve años y más de edad, y niñas desescolarizadas de nueve a diecisiete años en el territorio nacional. Colombia: Ministerio de Salud y Protección Social; 2014. Disponible en: https:// www.minsalud.gov.co/sites/rid/Lists/BibliotecaDigital/ RIDE/DE/DIJ/Circular-externa-0062-de-2014.pdf

46. Nogueira-Rodrigues A, Bukowski A, Paulino E, St. Louis J, Barrichello A, Sternberg C, et al. An alert to Latin America: Current human papillomavirus vaccination trends highlight key barriers to successful implementation. Cancer. 2017;123(12):2193-9.

47. Sánchez-Gómez LH, Yepes-Lujan FJ, HernándezFlórez LJ. La vacunación contra el virus del papiloma humano en Colombia. GRPS. 2001;13(27). Disponible en: https://revistas.javeriana.edu.co/index.php/ gerepolsal/article/view/12075

48. Reina JC, Muñoz N. Vaccine against human papilloma virus. Colomb Med (Cali). 2014;45(3):94-5. 
CONTRIBUCIÓN DE LOS AUTORES

Francisco Palencia-Sánchez: concepción de la revisión y del desarrollo metodológico, búsqueda y revisión de artículos, extracción de la información, redacción y revisión del documento.
Sandra Johanna Echeverry-Coral: concepción de la revisión y del desarrollo metodológico, búsqueda y revisión de artículos, extracción de la información, redacción y revisión del documento. 


\section{Anexo 1. \\ Estrategias de búsqueda}

\begin{tabular}{|c|c|}
\hline $\begin{array}{c}\text { Base de } \\
\text { datos }\end{array}$ & Estrategia de búsqueda empleada \\
\hline \multirow{4}{*}{ SCOPUS } & $\begin{array}{l}\text { TITLE-ABS-KEY ( ( "human papillomavirus vaccine" OR "gardasil” OR "quadrivalent hpv vaccine" } \\
\text { OR "hpv" OR “4HPV" OR "4hpv" OR "hpv) and ( impact or effectivenes or epidemiological and } \\
\text { monitoring or population and surveillance or program and evaluation or prevalence ) ) and } \\
\text { (" colombia " for " colombian AND population ) ) AND ( EXCLUDE ( SUBJAREA, "VETE “) ) }\end{array}$ \\
\hline & $\begin{array}{l}\text { TITLE-ABS-KEY ( ( "human papillomavirus vaccine" OR "Gardasil" OR "quadrivalent hpvvaccine" } \\
\text { OR "qHPV" OR "4HPV" OR "4VHPV" OR "HPV4" ) AND ( impact OR effectiveness OR "epi- } \\
\text { demiological monitoring" OR "population surveillance" OR "program evaluation" OR prevalence ) } \\
\text { AND ( "Colombia" ) OR ( "Colombian population") ) }\end{array}$ \\
\hline & TITLE-ABS-KEY ( ( "human papillomavirus vaccin*” AND “Colombia” ) ) \\
\hline & $\begin{array}{l}\text { TITLE-ABS-KEY ( ("human papillomavirus vaccine" OR "Gardasil" OR "quadrivalent hpv vaccine" } \\
\text { OR "qHPV" OR "4HPV" OR "4VHPV" OR "HPV4" ) AND ( "Colombia" ) OR ( "Colombian } \\
\text { population") ) }\end{array}$ \\
\hline \multirow{6}{*}{ BVS } & $\begin{array}{l}\text { (tw:(human papillomavirus vaccine L1, type 6,11,16,18)) AND (tw:(population surveillance)) AND } \\
\text { (tw:(Colombia)) }\end{array}$ \\
\hline & (tw:(human papillomavirus vaccine L1, type 6,11,16,18)) AND (tw:(prevalence)) AND (tw:(Colombia)) \\
\hline & $\begin{array}{l}\text { (tw:(vacuna cuadrivalente contra el virus del papiloma humano (tipo 6,11,16,18))) AND } \\
\text { (tw:(Colombia)) }\end{array}$ \\
\hline & $\begin{array}{l}\text { (tw:(vacuna tetravalente recombinante contra el virus del papiloma humano tipos 6,11,16,18)) AND } \\
\text { (tw:(Colombia)) }\end{array}$ \\
\hline & $\begin{array}{l}\text { (tw:(vacuna tetravalente recombinante contra el virus del papiloma humano tipos 6,11,16,18)) AND } \\
\text { (tw:(prevalencia)) AND (tw:(Colombia)) }\end{array}$ \\
\hline & (tw:(vacuna vph)) AND (tw:(Colombia)) \\
\hline \multirow{4}{*}{ Pubmed } & $\begin{array}{l}\text { ("human papillomavirus vaccine L1, type 6,11,16,18"[All Fields] OR "gardasil”[All Fields]) AND } \\
\text { ("Colombia" OR "Colombian" OR "Colombian population"[all fields]) }\end{array}$ \\
\hline & $\begin{array}{l}\text { ("quadrivalent hpv vaccine"[all fields] OR “qHPV” [all fields] OR "HPV4" [all fields] OR " } 4 \mathrm{HPV} \text { " [all } \\
\text { fields] OR "4VHPV"[all fields] AND ("Colombia" OR "Colombian" OR "Colombian population" [all } \\
\text { fields])) }\end{array}$ \\
\hline & 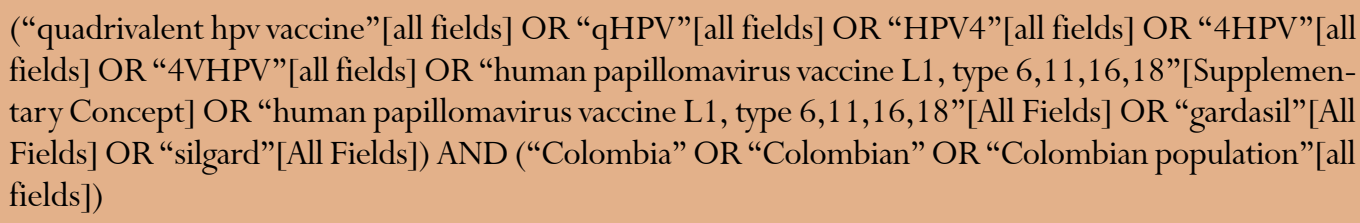 \\
\hline & 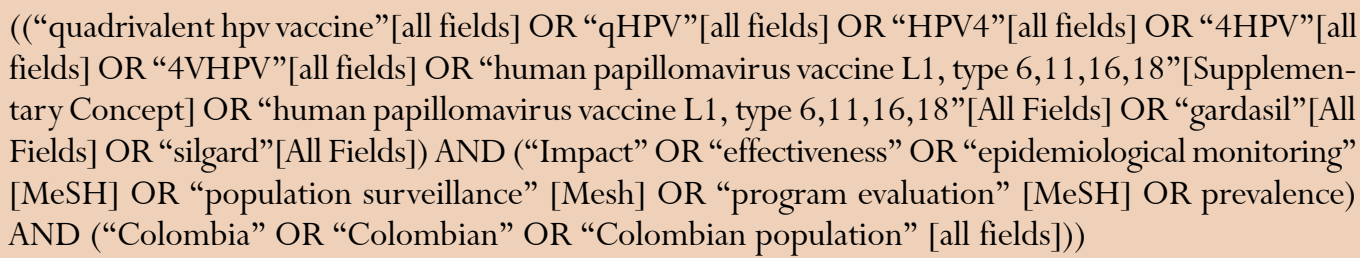 \\
\hline
\end{tabular}




\begin{tabular}{|c|c|}
\hline $\begin{array}{l}\text { Base de } \\
\text { datos }\end{array}$ & Estrategia de búsqueda empleada \\
\hline \multirow{3}{*}{$\begin{array}{l}\text { Web of } \\
\text { Science }\end{array}$} & $\begin{array}{l}((((\mathrm{TS}=((\text { Human }) \quad(\text { papillomavirus })(\text { vaccin } *))) \text { OR }(\mathrm{TS}=(\text { gardasil }))) \text { OR }(\mathrm{TS}=((\text { quadrivalent }) \\
(\mathrm{hpv})(\text { vaccine })))) \text { OR }(\mathrm{TS}=(\mathrm{hpv}))) \text { OR }((\mathrm{TS}=(4 \mathrm{hpr})))) \text { AND }(\mathrm{TS}=(\text { Colombia })) \\
\text { Indexes }=\text { SCI-EXPANDED, SSCI, A\&HCI, ESCI Timespan=All years }\end{array}$ \\
\hline & $\begin{array}{l}\text { TITLE: (human papillomavirus vaccin*) AND TITLE: (Colombia*) } \\
\text { Indexes=SCI-EXPANDED, SSCI, A\&HCI, ESCI Timespan=All years }\end{array}$ \\
\hline & $\begin{array}{l}((((\mathrm{TS}=((\text { Human }) \quad(\text { papillomavirus })(\text { vaccin } *))) \text { OR }(\mathrm{TS}=(\text { gardasil }))) \text { OR }(\mathrm{TS}=((\text { quadrivalent }) \\
(\mathrm{hpv})(\text { vaccine })))) \text { OR }(\mathrm{TS}=(\mathrm{hpv}))) \text { OR }((\mathrm{TS}=(4 \mathrm{hpr})))) \text { AND }(\mathrm{TS}=(\text { Colombia } *)) \\
\text { Indexes }=\text { SCIELO Timespan=All years }\end{array}$ \\
\hline \multirow{3}{*}{ OVID } & ("Human papillomavirus vaccine" and "Colombia").mp. [mp=title, abstract, full text, caption text $]$ \\
\hline & (human papillomavirus vaccination and Colombia).mp. $[\mathrm{mp}=$ title, abstract, full text, caption text $]$ \\
\hline & (gardasil and Colombia).mp. [mp=title, abstract, full text, caption text $]$ \\
\hline \multirow[b]{3}{*}{ EMBASE } & $\begin{array}{l}\text { ('wart virus vaccine':ti,ab,kw OR 'human papillomavirus vaccine } 11 \text { type } 61116 \text { 18':ti,ab,kw OR } \\
\text { 'human papillomavirus vaccine } 11 \text { type } 16 \text { 18':ti,ab,kw OR ‘human papillomavirus vaccination':ti,ab,kw } \\
\text { OR 'human papillomavirus type } 18 \text {-transformed cell line':ti,ab,kw) AND ‘colombia*':ti,ab,kw }\end{array}$ \\
\hline & $\begin{array}{l}\text { female'/exp AND ('colombia'/exp OR 'colombia' OR 'columbia') AND ('wart virus vaccine'/exp } \\
\text { OR 'human papilloma virus vaccine' OR 'human papillomavirus vaccine' OR 'papillomavirus vacci- } \\
\text { ne' OR 'papillomavirus vaccines' OR 'wart virus vaccine' OR 'ceravix' OR 'cervarix' OR 'gardasil' } \\
\text { OR 'gardasil 9' OR ‘human papillomavirus recombinant vaccine quadrivalent, types } 6,11,16,18 \text { ' } \\
\text { OR 'human papillomavirus type } 16 \text { vaccine' OR 'human papillomavirus vaccine (types } 16,18 \text { )' OR } \\
\text { 'human papillomavirus vaccine (types } 6,11,16,18 \text { ), recombinant, adsorbed' OR 'papilloma virus } \\
\text { vaccine' OR 'silgard') }\end{array}$ \\
\hline & $\begin{array}{l}\text { ('female'/exp OR 'female') AND ('colombia'/exp OR 'colombia') AND ('human papilloma virus va- } \\
\text { ccine'/exp OR 'human papilloma virus vaccine' OR 'human papillomavirus vaccine'/exp OR 'human } \\
\text { papillomavirus vaccine' OR 'papillomavirus vaccine'/exp OR 'papillomavirus vaccine' OR 'papilloma- } \\
\text { virus vaccines'/exp OR 'papillomavirus vaccines' OR 'wart virus vaccine'/exp OR 'wart virus vaccine' } \\
\text { OR 'ceravix'/exp OR 'ceravix' OR 'cervarix'/exp OR 'cervarix' OR 'gardasil'/exp OR 'gardasil' OR } \\
\text { 'gardasil 9'/exp OR 'gardasil 9' OR 'human papillomavirus recombinant vaccine quadrivalent, types } \\
6,11,16,18 \text { '/exp OR 'human papillomavirus recombinant vaccine quadrivalent, types } 6,11,16 \text {, } \\
18 \text { ' OR 'human papillomavirus type } 16 \text { vaccine'/exp OR 'human papillomavirus type } 16 \text { vaccine' } \\
\text { OR 'human papillomavirus vaccine (types } 16,18) \text { '/exp OR 'human papillomavirus vaccine (types } \\
16,18 \text { )' OR 'human papillomavirus vaccine (types } 6,11,16,18 \text { ), recombinant, adsorbed'/exp OR } \\
\text { 'human papillomavirus vaccine (types } 6,11,16,18), \text { recombinant, adsorbed' OR 'papilloma virus } \\
\text { vaccine'/exp OR 'papilloma virus vaccine' OR 'silgard'/exp OR 'silgard') }\end{array}$ \\
\hline \multirow{5}{*}{$\begin{array}{l}\text { Google } \\
\text { Scholar } \\
\text { (Literatura } \\
\text { Gris) }\end{array}$} & (papilloma) OR (HPV) AND (vaccine) AND (adverse) AND (Colombia) AND (Colombian population) \\
\hline & $\begin{array}{l}\text { (papilloma) OR (HPV) AND (vaccine) AND (adverse events) AND (Colombia) AND (Colombian } \\
\text { population) }\end{array}$ \\
\hline & $\begin{array}{l}\text { (papilloma) OR (HPV) AND (vaccin*) AND (adverse events) AND (Colombia) AND (Colombian } \\
\text { population) }\end{array}$ \\
\hline & (papiloma) OR (VPH) AND (vacuna) AND (adversos) AND (Colombia) AND (población colombiana) \\
\hline & $\begin{array}{l}\text { (papiloma) OR (VPH) AND (vacuna) AND (eventos adversos) AND (Colombia) AND (población } \\
\text { colombiana) }\end{array}$ \\
\hline
\end{tabular}




\begin{tabular}{|c|c|}
\hline $\begin{array}{c}\text { Base de } \\
\text { datos }\end{array}$ & Estrategia de búsqueda empleada \\
\hline \multirow{5}{*}{$\begin{array}{c}\text { Google } \\
\text { Scholar } \\
\text { Literatura } \\
\text { Gris) }\end{array}$} & (human papillomavirus vaccine $\mathrm{L} 1$, type $6,11,16,18$ ) AND (Colombia) or (Colombian population) \\
\hline & $\begin{array}{l}\text { (human papillomavirus vaccine L1, type } 6,11,16,18 \text { ) AND (Adverse events) AND (Colombia) or } \\
\text { (Colombian population) }\end{array}$ \\
\hline & (quadrivalent hpv vaccine) AND (Colombia) or (Colombian population) \\
\hline & (quadrivalent hpv vaccine) AND (adverse events) AND (Colombia) or (Colombian population) \\
\hline & (Human papillomavirus vaccine) AND (adverse events) AND (Colombia) or (Colombian population) \\
\hline
\end{tabular}

